\title{
Diagnosis and Monitoring of Chronic Myeloid Leukemia: Chiang Mai University Experience
}

\author{
Adisak Tantiworawit ${ }^{1}$, Supanat Kongjarern ${ }^{2}$, Ekarat Rattarittamrong ${ }^{1 *}$, \\ Suree Lekawanvijit ${ }^{3}$, Kanokkan Bumroongkit ${ }^{4}$, Nonglak Boonma ${ }^{4}$, Thanawat \\ Rattanathammethee ${ }^{1}$, Sasinee Hantrakool ${ }^{1}$, Chatree Chai-Adisaksopha ${ }^{1}$, Lalita \\ Norasetthada $^{1}$
}

\begin{abstract}
Background: A diagnosis of chronic myeloid leukemia (CML) is made on discovery of the presence of a Philadelphia (Ph) chromosome. The success of the treatment of this form of leukemia with tyrosine kinase inhibitor (TKI) is monitored by reduction of the Ph chromosome. Objective: To compare the role of conventional cytogenetic (CC) methods with a real time quantitative polymerase chain reaction (RQ-PCR) and fluorescence in situ hybridization (FISH) for diagnosis and treatment monitoring of CML patients. The secondary outcome was to analyze the treatment responses to TKI in CML patients. Materials and Methods: This was a retrospective study of CML patients who attended the Hematology clinic at Chiang Mai University Hospital from 2005-2010. Medical records were reviewed for demographic data, risk score, treatment response and the results of CC methods, FISH and RQ-PCR. Results: One hundred and twenty three cases were included in the study, 57.7\% of whom were male with a mean age of 46.9 years. Most of the patients registered as intermediate to high risk on the Sokal score. At diagnosis, 121 patients were tested using the $\mathrm{CC}$ method and 118 (95.9\%) were identified as positive. Five patients failed to be diagnosed by $\mathrm{CC}$ methods but were positive for BCR-ABL1 using the FISH method. Imatinib was the first-line treatment used in 120 patients $(97.6 \%)$. In most patients (108 out of 122 , 88.5\%), a complete cytogenetic response (CCyR) was achieved after TKI therapy and in 86 patients $(70.5 \%)$ CCyR was achieved long term by the $\mathrm{CC}$ method. Five out of the 35 analyzed patients in which $\mathrm{CCyR}$ was achieved by the CC method had a positive FISH result. Out of the 76 patients in which CCyR was achieved, RQ-PCR classified patients to only CCyR in 17 patients $(22.4 \%)$ with a deeper major molecular response (MMR) in 4 patients $(5.3 \%)$ and complete molecular response (CMR) in 55 patients $(\mathbf{7 2 . 4 \%})$. In the case of initial therapy, CCyR was achieved in 95 patients $\mathbf{( 7 9 . 1 \% )}$ ) who received imatinib and in both patients who received dasatinib $(\mathbf{1 0 0 \%})$. For the second line treatment, nilotinib were used in 30 patients and in 19 of them $(63.3 \%)$ CCyR was achieved. In half of the 6 patients $(50 \%)$ who received dasatinib as second line or third line treatment CCyR was also achieved. Conclusions: CML patients had a good response to TKI treatment. FISH could be useful for diagnosis in cases where $\mathrm{CC}$ analysis failed to detect the Ph chromosome. RQ-PCR was helpful in detecting any residual disease and determining the depth of the treatment response at levels greater than the $\mathrm{CC}$ methods.
\end{abstract}

Keywords: Chronic myeloid leukemia - conventional cytogenetic method - FISH - Philadelphia chromosome

Asian Pac J Cancer Prev, 17 (4), 2159-2164

\section{Introduction}

Chronic myeloid leukemia(CML) is a myeloproliferative neoplasm (MPN) which is characterized by the presence of leukocytosis with many stages of differentiation of neutrophils. It has a distinct pathogenesis due to the presence of the BCR-ABL1 fusion gene or a Philadelphia $(\mathrm{Ph})$ chromosome resulting from reciprocal translocation of chromosome 9 and 22 [t $(9 ; 22)(\mathrm{q} 34 ; \mathrm{q} 11)]$ (Vardiman et al., 2008). CML is classified into the chronic phase (CP), accelerated phase (AP), and blastic phase (BP) according to the number of blast cells in the blood or bone marrow (BM), degree of basophilia, presence of persistent thrombocytopenia, response to therapy, clonal chromosomal abnormalities in $\mathrm{Ph}+$ cells, and presence of extramedullary blast proliferation (Vardiman et al., 2008; Baccarani et al., 2013).

The diagnosis of CML can be obtained by conventional

${ }^{1}$ Division of Hematology, Department of Internal Medicine, ${ }^{3}$ Department of Pathology, ${ }^{4}$ Department of Anatomy, Faculty of Medicine, Chiang Mai University, Chiang Mai, ${ }^{2}$ Department of Internal Medicine, Lampang Hospital, Lampang, Thailand *For correspondence: ekarat_r@hotmail.com 
cytogenetic (CC) analysis to demonstrate the presence of the $\mathrm{Ph}$ chromosome. However, it has limitations in cases of variant translocation or cryptic translocation. In these situations, the presence of BCR-ABL1 fusion genes or transcripts by fluorescence in situ hybridization (FISH) analysis or reverse transcriptase polymerase chain reaction (RT-PCR) or real-time quantitative PCR (RQ-PCR) can be used for the diagnosis (Vardiman et al., 2008; Baccarani et al., 2013).

Before tyrosine kinase inhibitor (TKI) was available, allogeneic hematopoietic stem cell transplantation (alloSCT) was widely used as a curative treatment but it had limitations including treatment-related mortality, availability of matched donors, age, and physical status of the patients. As a result, the therapy for CML was mainly oral chemotherapy such as hydroxyurea which could induce a hematologic response (HR) but not a cytogenetic response $(\mathrm{CyR})$. Therefore, patients who received this treatment eventually died due to progression to BP at a median survival at about 4 years (Vardiman et al., 2008).

The first TKI that changed the paradigm of therapy for CML is imatinib. A phase 3 International Randomized Study of Interferon and STI571 (IRIS) showed that this targeted therapy can induce complete HR (CHR) and complete CyR (no Ph chromosome detected; CCyR) in $95.3 \%$ and $76.2 \%$ of CML-CP patients, respectively (O'Brien et al., 2003). For long-term outcomes, the best observed rate of CCyR was $82 \%$ and the 7 -year event-free survival (EFS) and overall survival (OS) were $81 \%$ and $86 \%$, respectively (O'Brien et al., 2008). These figures were higher than historical allo-SCT data (Gratwohl et al., 2006). As a result, TKI became the standard treatment for CML-CP. The second generation TKIs, nilotinib and dasatinib, were investigated rapidly and showed efficacy in both front-line therapy (Saglio et al., 2010 and Kantarjian et al., 2010) and second-line therapy in cases of imatinib intolerance or resistance (Giles et al., 2013; Shah et al., 2014).

Treatment with TKIs is initially monitored by physical examination and normalization of complete blood count (CBC) to determine HR. Apart from this, the reduction of the $\mathrm{Ph}$ chromosomes should be monitored by $\mathrm{CC}$ methods (CyR) or RQ-PCR for BCR-ABL1 transcript levels [molecular response (MR)] (Baccarani et al., 2013).

The primary objective of this study was to compare the role of the CC methods with RQ-PCR and FISH for diagnosis and treatment monitoring of CML patients. The secondary objective was to analyze the treatment responses of CML patients in Chiang Mai University Hospital.

\section{Materials and Methods}

\section{Study overview}

This study was approved by the Institutional Review Board (IRB) of the Faculty of Medicine, Chiang Mai University, Thailand and performed at Maharaj Nakorn Chiang Mai Hospital. All CML-CP patients aged more than 15 years who attended the Hematology Clinic from $1^{\text {st }}$ January 2005 to $31^{\text {st }}$ December 2010 were retrospectively reviewed for demographic data, CML risk score including
Sokal score (Sokal et al., 1984), Euro score (Hasford et al., 1998), EUTOS score (Hasford et al., 2011), and treatment response. The criteria of treatment responses including $\mathrm{HR}, \mathrm{CyR}$, and MR were defined according to European LeukemiaNet (ELN) criteria (Baccarani et al., 2009; Baccarani et al., 2013). The results of the CC methods, FISH and RQ-PCR in each patient at diagnosis and during follow-up after TKI therapy were also analyzed. SPSS statistical program version 16.0 was used for data analysis.

\section{Conventional cytogenetic (CC) analysis}

Metaphase chromosomes were prepared from 0.25$0.5 \mathrm{ml}$ of bone morrow. The samples were cultured in $10 \mathrm{ml}$ of RPMI 1640 medium containing $20 \%$ fetal calf serum, including $0.015 \mu \mathrm{g} / \mathrm{ml}$ colchicine, $6 \mathrm{mg} /$ $\mathrm{ml}$ deoxythymidine, ampicillin and streptomycin. The cultures were incubated at $37^{\circ} \mathrm{C}$ for 24 hours and after that $0.1 \mu \mathrm{g} / \mathrm{ml}$ colchicine were added. The cultures were incubated at $37^{\circ} \mathrm{C}$ for a further 30 minutes. The chromosomes were harvested using conventional methods. Briefly, the bone marrow cultures were washed with phosphate buffer saline (PBS) and then exposed to $0.075 \mathrm{M} \mathrm{KCl}$ for hypotonic treatment (3 times), methanol: acetic acid (3:1) was used for fixation of cell membranes (3 times). Cells were dropped onto microscopic slides and kept overnight at room temperature. Chromosome spreads were stained with G- or Q-banding techniques. Ten metaphase chromosomes were analyzed for diagnosis and 20 metaphase chromosomes for follow up.

\section{Fluorescence in situ hybridization (FISH) analysis}

FISH analysis for the $\mathrm{t}(9 ; 22)(\mathrm{q} 34 ; \mathrm{q} 11.2)$ were detected on both bone marrow interphase nuclei or metaphase chromosomes spreads prepared using CC methods. Slides were treated with $0.1 \mu \mathrm{g} / \mu \mathrm{l}$ RNase solution and $0.01 \%$ pepsin followed by $1 \%$ formaldehyde/1M MgCl in PBS and dehydrated in a series of ethanols. ALSI BCR/ABL1 dual color dual fusion probe (Vysis, Downers Grove, Illinois, USA) was used according to the manufacturer's instructions with slight modification. Briefly, the slide was incubated in denaturing solution ( $70 \%$ formamide/2XSSC) at $73^{\circ} \mathrm{C}$ for 10 minutes, followed by dehydration in a series of ethanols. Probe mixture denaturation was carried out at $73^{\circ} \mathrm{C}$ for 5 minutes. Ten microliters of the probe mixture were hybridized to a $22 \times 22 \mathrm{~mm}$ area of slides for 16-20 hours at $37^{\circ} \mathrm{C}$ in a humidified chamber and the hybridized area was sealed with fixogum. Slides were washed in $0.4 \mathrm{X}$ $\mathrm{SSC} / 0.3 \% \mathrm{NP}-40$ at $73^{\circ} \mathrm{C}$ for 2 minutes followed by $2 \mathrm{X}$ $\mathrm{SSC} / 0.1 \% \mathrm{NP}-40$ at room temperature for 1 minute. Slides were then dehydrate in a series of ethanols and were then counterstained with $0.5 \mu \mathrm{g} / \mathrm{ml}$ of DAPI for 4 minutes.

Evaluation of the FISH signals, 200 interphase nuclei and metaphase chromosomes were performed under 1000x magnification using an Axioskop 2 fluorescence microscope (Carl Zeiss, Gottingen, Germany) and ISIS software (Meta Systems, GmBH, Altlussheim, Germany). Cells with normal chromosome 9 and 22 show two separate signals of each orange and green color. While cells containing $t(9 ; 22)$ show one red signal from normal chromosome 9 and one green signal from normal chromosome 22 and two orange/green fusion signals from 
the derivative 9 and 22 chromosomes.

\section{$R T-P C R$ and $R Q-P C R$ for BCR-ABL1 transcripts}

Subtypes of the BCR-ABL1 transcripts (b2a2, b3a2) were determined by RT-PCR before quantitative analysis by RQ-PCR. Total RNA was extracted from 10 mL EDTA-blood using trizol method (Invitrogen, CA, USA). Two $\mu \mathrm{g}$ of total RNA was reverse-transcribed to cDNA with SuperScript ${ }^{\circledR}$ III First-Strand Synthesis System (Invitrogen, CA, USA). For RT-PCR, $2 \mu \mathrm{L}$ of cDNA was amplified using sequence-specific primers (Invitrogen, CA, USA) for p210 BCR-ABL transcripts. Glyceraldehyde-3-phosphate dehydrogenase (GAPDH) which was used as an endogenous control gene was amplified in separate reaction. Primers used for RT-PCR were as follows: BCR-ABL (p210)-forward (F), 5'-GAT GCT GAC CAA CTC GTG TG-3'; BCR-ABL (p210)reverse (R), 5'-AAC GAA AAG GTT GGG GTC AT-3'; GAPDH-F, 5'-ACC ACA GTC CAT GCC ATC AC-3' and GAPDH-R; 5'-TCC ACC ACC CTG TTG CTG TA3'. PCR reactions were performed in $25 \mu$ l total volume with $5 \mathrm{mM} \mathrm{MgCl}_{2}, 0.2 \mathrm{mM}$ dNTP mixture, $0.32 \mu \mathrm{M}$ each primer and 1 unit of Taq DNA Polymerase (Invitrogen, CA, USA). The cycle conditions were as follows: an initial denaturation at $95^{\circ} \mathrm{C}$ for 5 minutes, followed by 40 cycles of $95^{\circ} \mathrm{C}$ for $30 \mathrm{sec}, 58^{\circ} \mathrm{C}$ for $30 \mathrm{sec}$ and $72^{\circ} \mathrm{C}$ for $45 \mathrm{sec}$. PCR products were loaded onto $2 \%$ agarose gel for gel electrophoresis before being stained with ethidium bromide. The 194-bp, 265-bp and 400-bp PCR products represented b2a2, b3a2 and GAPDH, respectively.

For RQ-PCR using Applied Biosystems 7500 Fast Real Time PCR System, $2 \mu \mathrm{L}$ of cDNA was amplified using the same sequence-specific primers (Invitrogen, CA, USA) as RT-PCR and TagManfluorogenic probe FAMAGA CCC TGA GGC TCA AAG TCA GAT GCT ACTTAMRA (AITbiotech, Singapore) for p210 BCR-ABL transcripts. ABL was used as an endogenous control to correct for the expression of $\mathrm{p} 210 \mathrm{BCR}-\mathrm{ABL}$ genes. ABL primers (Invitrogen, CA, USA) and probe (AITbiotech, Singapore) were as follows: ABL-F, 5'-GCC TCA GGG TCT GAG TGAAG-3'; ABL-R, 5'-ACA CCA TTC CCC ATT GTG AT-3'; ABL-probe, FAM-AGA GTG TTA TCT CCA CTG GCC ACA AAA TCA-TAMRA. Plasmid cDNAs of p210 BCR-ABL and ABL were employed as a standard at concentrations of $10^{2}, 10^{3}, 10^{4}, 10^{5}$ and $10^{6}$ copies per $\mu \mathrm{l}$. PCR reactions were performed in $20 \mu \mathrm{l}$ total volume with $0.32 \mu \mathrm{M}$ each primer, $0.1 \mu \mathrm{M}$ probe and 1xPCR TaqMan Gene Expression Master Mix (Life Technologies, CA, USA). The cycle conditions were as follows: an initial denaturation at $95^{\circ} \mathrm{C}$ for 10 minutes, followed by 50 cycles of $95^{\circ} \mathrm{C}$ for $15 \mathrm{sec}, 58^{\circ} \mathrm{C}$ for $15 \mathrm{sec}$ and $72^{\circ} \mathrm{C}$ for $30 \mathrm{sec}$.

\section{Results}

\section{Demographic data}

There were 190 CML patients during the study period but 67 patients were excluded due to being lost to followup or were referred to follow-up at other hospitals. Finally, 123 patients were included in the study. Table 1 shows the clinical characteristics of the CML patients included in this study. Seventy-one patients $(57.7 \%)$ were male with a mean age of 46.9 years (range 17-78). Imatinib was the most prescribed TKI as the first-line therapy (120 patients, $97.6 \%)$. Two patients (1.6\%) received dasatinib and one patient didn't take TKI due to economic problems.

Most patients were classified as high risk and intermediate risk according to their Sokal score $(41.5 \%$ and $39.8 \%$, respectively) and intermediate risk by Euro score $(48.8 \%)$. However, when EUTOS score was used, most of the patients were categorized as low risk (78.9\%).

\section{Laboratory diagnosis of CML}

From the CC analyses, 118 patients (95.9\%) were correctly diagnosed with CML. The remaining 3 patients $(2.4 \%)$ had no metaphase and 2 patients $(1.6 \%)$ underwent bone marrow (BM) aspiration and $\mathrm{CC}$ analyses from other hospitals with negative results.

FISH analyses were performed on 20 patients at diagnosis and BCR-ABL1 fusion genes were detected in all cases including 5 patients who were not diagnosed by CC methods. RT-PCR and RQ-PCR were not used for the diagnosis of CML in this study on any patients.

\section{Laboratory monitoring after TKI therapy}

$\mathrm{CC}$ methods were used in 122 patients who received TKI in order to monitor response. CCyR was achieved in 108 patients $(88.5 \%)$ with a median time to CCyR of 12.9 months. Eighty-six patients $(70.5 \%)$ remained in continuous CCyR by CC method at the last follow up date.

There were 35 patients who had FISH analyses performed for monitoring and 10 patients (28.5\%) remained positive for BCR-ABL1 fusion genes. Table 2 compares FISH analysis and $\mathrm{CC}$ analysis for monitoring of CML patients. FISH analyses led to the successful detection of BCR-ABL1 fusion genes in 1 patient who had no metaphase and 5 patients who did not have any $\mathrm{Ph}$ chromosomes detected by $\mathrm{CC}$ methods. On the contrary, 2 patients $(5.7 \%)$ who had negative BCR-ABL1 fusion genes discovered by FISH were positive for $\mathrm{Ph}$

Table 1. Clinical Characteristics of CML Patients in the Study

\begin{tabular}{llc}
\hline \multicolumn{2}{c}{ Clinical characteristics } & N (Total=123) \\
\hline \multirow{2}{*}{ Sex } & Male & $71(57.7 \%)$ \\
& Female & $52(42.3 \%)$ \\
Age (Year) & Mean \pm SD & $46.9 \pm 17.8$ \\
& Min/Max & $17 / 78$ \\
Medication: & None & $1(0.81 \%)$ \\
First line & Imatinib & $120(97.6 \%)$ \\
& Dasatinib & $2(1.6 \%)$ \\
& Nilotinib & $0(0 \%)$ \\
& Sokal (Mean \pm SD) & $2.9 \pm 9.9$ \\
& High risk & $51(41.5 \%)$ \\
& Intermediate risk & $49(39.8 \%)$ \\
Clinical risk & Low risk & $23(18.7 \%)$ \\
score & Euro (Mean \pm SD) & $1181.2 \pm 662.1$ \\
& High risk & $31(25.20 \%)$ \\
& Intermediate risk & $60(48.78 \%)$ \\
& Low risk & $32(26.02 \%)$ \\
& EUTOS (Mean \pm SD) & $62.1 \pm 44.0$ \\
& High risk & $26(21.14 \%)$ \\
& Low risk & $97(78.86 \%)$ \\
\hline
\end{tabular}


Table 2. Comparison Between Conventional Cytogenetics Analysis (CC) and Fluorescence in Situ Hybridization (FISH) Analysis for Monitoring of 35 CML Patients

\begin{tabular}{|cccc|}
\hline $\begin{array}{c}\text { Conventional } \\
\text { cytogenetics (CC) }\end{array}$ & $\begin{array}{c}\text { Fluorescence in } \\
\text { situ hybridization } \\
\text { (FISH) }\end{array}$ & $\mathrm{N}=35$ & $\%$ \\
\hline Positive & Positive & 4 & 11.4 \\
Positive & Negative & 2 & 5.7 \\
Negative & Positive & 5 & 14.3 \\
Negative & Negative & 18 & 51.4 \\
No interpretation & Positive & 1 & 2.9 \\
No interpretation & Negative & 5 & 14.3 \\
\hline
\end{tabular}

chromosomes when CC methods were used.

RQ-PCR was carried out for the monitoring of 103 patients. This method could determine the depth of response into BCR-ABL1 transcripts $<10 \%$ which was comparable with major $\mathrm{CyR}$ (Ph chromosome $<35 \%$; MCyR) by CC methods, BCR-ABL1 transcripts $<1 \%$ that correspond with CCyR, BCR-ABL1 transcript $<0.1 \%$ or major MR (MMR), and undetected BCR-ABL1 transcript or complete MR (CMR). Analysis of 76 patients in whom CCyR was achieved who also had RQ-PCR monitoring showed CMR in most patients [55 patients (72.4\%)] followed by CCyR [17 patients (22.3\%)] and MMR [4 patients $(5.3 \%)$ ].

\section{Response to TKI therapy}

As describes previously, CCyR was achieved in 108 patients out of 122 TKI-treated patients (88.5\%) after TKI therapy including 84 patients treated with imatinib, 19 patients with nilotinib, and 5 patients with dasatinib.

In the 120 patients who received imatinib as a first-line therapy, 95 (79.1\%) of them showed initial achievement of CCyR. However, 11 patients (9.2\%) eventually showed loss of CCyR in addition to another 25 (20.8\%) patients who failed to have any CCyR. Overall 84 patients (70\%) had continuous CCyR. In cases of failure, imatinib was switched to nilotinib and dasatinib in 30 and 3 patients, respectively. Nineteen patients $(63.3 \%)$ who received nilotinib demonstrated CCyR both in patients who did not show any CCyR (15 patients) and patients that later showed a loss of CCyR (4 patients). The majority of them also showed CMR (10 patients) and MMR (5 patients).

Dasatinib as second-line therapy was prescribed for 2 patients who did not show any CCyR and 1 patient with a loss of CCyR after imatinib. All patients finally showed CCyR. Two imatinib and nilotinib failure patients subsequently received dasatinib but only one then achieved CCyR. Both patients who received dasatinib as first-line therapy showed CCyR and CMR.

\section{Discussion}

This study provides information about demographic data, laboratory diagnosis, monitoring and treatment responses of CML patients in Thailand. There was a slight male predominance of CML patients in this study with a mean age of 47 years. This age was equivalent to a study carried out in Iran (Payandeh et al., 2015) but higher than a Chinese ENEST study (39 years in imatinib group and 41 years in nilotinib group) (Wang et al., 2015). In a large prospective IRIS study (O'Brien et al, 2003), a greater number of patients were also male with a median age of 50 years, slightly higher than this study. The age range did not largely differ between the two studies (17-78 years in this study vs 17-70 years in IRIS study). However, the clinical risk scores of the patients in this study were mainly intermediate to high risk according to Sokal and Euro scores compared to low to intermediate risk in the IRIS study. This may be explained by a lack of knowledge, health check-up and awareness of CML in Thai people which consequently led to relatively late presentation.

This study confirmed that diagnosis of CML by $\mathrm{CC}$ methods had some limitations and may result in a critical delay of treatment. Three patients who had no metaphase and two patients who had negative results from this method could have had an established diagnosis by using the FISH analysis which has greater sensitivity (Kantarjian et al, 2008). Nevertheless, CC analysis remains the recommended method (Baccarani et al., 2013) since it is available in many hospitals and it can establish the diagnosis in more than $95 \%$ of patients as shown in this study. Moreover, it can demonstrate additional chromosomal abnormalities which influence the prognosis (Fabarius et al., 2011). More sensitive methods such as FISH or RT-PCR should be used in cases which have no metaphase or negative results from CC analysis but CML is still suspected (Vardiman et al., 2008).

$\mathrm{CC}$ analysis is also the recommended method for monitoring response after TKI therapy (Baccarani et al., 2013). The patients in whom CCyR was achieved had good clinical outcomes including progression-free survival and overall survival and CCyR is accepted as the minimal goal of CML therapy (Baccarani et al., 2013). Likewise, for purposes of diagnosis, the $\mathrm{CC}$ method has the problem concerning the presence of metaphases after treatment. Before the era of RQ-PCR monitoring, FISH analysis was investigated with the aim of solving this problem. The interphase FISH was shown to detect the BCR-ABL1 fusion gene in $17.3 \%$ of CCyR patients by CC method (Testoni et al, 2009) when a cut-off of less than $1 \%$ positive nuclei was used. In this study, the FISH analysis was not only helpful in a case of no metaphase but also enabled the detection of any residual disease in cases with CCyR. However, its use was limited by a lack of standardization and studies about the prognostic values of residual diseases determined by the FISH method. ELN recommended that FISH of blood interphase cell nuclei could be substituted for the CC method only for the assessment of CCyR, which is defined by $<1 \% \mathrm{BCR}$ ABL1-positive nuclei out of at least 200 nuclei (Baccarani et al., 2013). Moreover, its sensitivity was questioned since about $5 \%$ of patients in this study who had negative BCR-ABL1 from FISH still had Ph chromosomes by $\mathrm{CC}$ methods as well as about $1.6 \%$ in the previous study (Testoni et al, 2009). FISH analysis was performed in the short period of time before RQ-PCR was available in our institution.

The RQ-PCR method for detection of BCR-ABL1 
transcript levels or "molecular response" is widely used as a monitoring tool in CML because of its sensitivity and availability of international standardization method (Branford et al., 2008). It is also a recommended method for monitoring in addition to CC analysis depending on local facilities and on the degree of molecular standardization at the local laboratory (Baccarani et al., 2013). In this study, RQ-PCR could identify CMR and MMR in $72.4 \%$ and $5.3 \%$ of CCyR patients determined by $\mathrm{CC}$ methods, respectively. A deeper response, such as MMR, is also a recommended target of BCR-ABL1 transcripts for CML therapy according to current ELN guidelines (Baccarani et al., 2013). The patients who reach this milestone by 18 months have a very low possibility of suffering from disease progression to $\mathrm{AP} / \mathrm{BP}$ as well as loss of CCyR (Hughes et al., 2010). In addition, a BCR-ABL1 transcript level $<10 \%$ at 3 months which corresponds to MCyR was reported to be prognostically significant in several studies (Hughes et al., 2010 and Marin D et al., 2012) but the role of a change of treatment according to this milestone is still under investigation (Baccarani et al., 2013; Yeung et al., 2015).

Regarding a more extensive response, CMR [currently substituted by the term "molecularly undetectable leukemia" (Baccarani et al., 2013)] or $\mathrm{MR}^{4.5}$ at 4 years was shown to be more predictive of long-term survival than CCyR (Hehlmann et al., 2013). In addition, about $40 \%$ of patients in whom CMR was achieved were found to have sustained remission even after TKI discontinuation trial (Mahon et al., 2010).

The role of RQ-PCR for BCR-ABL1 transcripts in the diagnosis were not evaluated in this study because this test was only recently available at Chiang Mai University. Therefore, it was used mainly for monitoring after TKI therapy especially in the patients who already were at the point of CCyR. However, there is some evidence that the baseline BCR-ABL1 transcripts may be useful in calculating the rate of decline or halving time of BCRABL1 transcript levels. Patients with a BCR-ABL1 halving time of less than 76 days also had good outcome even though they did not have early MR or BCR-ABL1 transcripts of less than $10 \%$ at 3 months (Branford et al., 2014).

Almost all patients in this study received imatinib as the front-line treatment although nilotinib and dasatinib were shown to induce more rapid and deeper response in the large randomized trials (Saglio G, et al., 2010 and Kantarjian $\mathrm{H}$ et al., 2010). This may be explained by national policy and economic status in Thailand as well as an emerging concern about long-term complications of both second-generation TKIs (Apperley, 2015). The patients who were given imatinib in whom $79.1 \%$ had CCyR were close to the successful outcomes in the IRIS study (76.2\%) (O'Brien et al., 2003) in spite of patients included in this study having higher risk scores. Meanwhile, the long-term follow-up and monitoring are warranted since in the IRIS study, only $57 \%$ of patients had maintained CCyR (O’Brien et al., 2008).

Both nilotinib and dasatinib were effective TKIs for the patients in cases of imatinib resistance and intolerance. In the present study, nilotinib could induce
CCyR in about $60 \%$ of cases as a second-line agent, a figure which was higher than the international phase II study of nilotinib after imatinib resistance or intolerance (Giles et al., 2013) (45\%) and Expanding Nilotinib Access in Clinical Trail (ENACT) study (34\%) (Nicolini et al., 2012). Only a few patients received dasatinib as first-, second-, or third-line in this study but in most of the patients who were given it there was a good response.

The limitation of this retrospective study was the limited numbers of CML patients who were analysed using FISH. The test was primarily performed in cases where CC methods showed no metaphase and its utility had declined after RT-PCR and RQ-PCR for the BCR-ABL1 method were available in the institution. No analysis of the correlation between response and time after TKI therapy as well as long-term outcomes such as treatment complications, progression-free survival and overall survival were carried out as they were out of the scope of this study.

In conclusions, FISH analysis is a sensitive method which can be used for the diagnosis of CML in cases where conventional cytogenetic analysis has failed to indicate a $\mathrm{Ph}$ chromosome or no metaphase. The RQ-PCR method provides greater sensitivity in detecting residual disease compared with CC methods. The treatment responses to TKI, the main one being imatinib, were not significantly different from studies carried out in Western countries.

\section{References}

Apperley JF (2015). Chronic myeloid leukemia. Lancet, 385, 1447-59.

Baccarani M, Cortes J, Pane F, et al (2009). Chronic myeloid leukemia: an update of concepts and management recommendations of European LeukemiaNet. J Clin Oncol, 27, 6041-51.

Baccarani M, Deininger MW, Rosti G, et al (2013). European LeukemiaNet recommendations for the management of chronic myeloid leukemia. Blood, 122, 872-84.

Branford S, Fletcher L, Cross NC, et al (2008). Desirable performance characteristics for BCR-ABL measurement on an international reporting scale to allow consistent interpretation of individual patient response and comparison of response rates between clinical trials. Blood, 112, 3330-8.

Branford S, Yeung DT, Parker WT, et al (2014). Prognosis for patients with CML and $>10 \%$ BCR-ABL1 after 3 months of imatinib depends on the rate of BCR-ABL1 decline. Blood, 124, 511-8.

Fabarius A, Leitner A, Hochhaus A, et al (2011). Impact of additional cytogenetic aberrations at diagnosis on prognosis of CML: long-term observation of 1151 patients from the randomized CML Study IV. Blood, 118, 6760-8.

Giles FJ, le Coutre PD, Pinilla-Ibarz J, et al (2013). Nilotinib in imatinib-resistant or imatinib-intolerant patients with chronic myeloid leukemia in chronic phase: 48-month follow-up results of a phase II study. Leukemia, 27, 107-12.

Gratwohl A, Brand R, Apperley J, et al (2006). Allogeneic hematopoietic stem cell transplantation for chronic myeloid leukemia in Europe 2006: transplant activity, long-term data and current results. An analysis by the chronic leukemia working party of the european group for blood and marrow transplantation (EBMT). Haematologica, 91, 513-21.

Hasford J, Pfirrmann M, Hehlmann R, et al (1998). A new prognostic score for survival of patients with chronic 
Adisak Tantiworawit et al myeloid leukemia treated with interferon alfa. J Natl Cancer Inst, 90, 850-8.

Hasford J, Baccarani M, Hoffmann V, et al (2011). Predicting complete cytogenetic response and subsequent progressionfree survival in 2060 patients with CML on imatinib treatment: the EUTOS score. Blood, 118, 686-92.

Hehlmann R, Muller MC, Lauseker M, et al (2013). Deep molecular response is reached by the majority of patients treated with imatinib, predicts survival, and is achieved more quickly by optimized high-dose imatinib: results from the randomized CML-Study IV. J Clin Oncol, 32, 415-23.

Hughes TP, Hochhaus A, Branford S, et al (2010). Long-term prognostic significance of early molecular response to imatinib in newly diagnosed chronic myeloid leukemia: an analysis from the International Randomized Study of Interferon and STI571 (IRIS). Blood, 116, 3758-65.

Kantarjian H, Sawyers C, Hochhaus A, et al (2002). Hematologic and cytogenetic responses to imatinib mesylate in chronic myelogenous leukemia. $N$ Engl J Med, 346, 645-52.

Kantarjian H, Schiffer C, Jones D, et al (2008). Monitoring the response and course of chronic myeloid leukemia in the modern era of BCR-ABL tyrosine kinase inhibitors: practical advice on the use and interpretation of monitoring methods. Blood, 111, 1774-80.

Kantarjian H, Shah NP, Hochhaus A, et al (2010). Dasatinib versus imatinib in newly diagnosed chronic-phase chronic myeloid leukemia. $N$ Engl J Med, 362, 2260-70.

Mahon FX, Rea D, Guilhot J, et al (2010). Discontinuation of imatinib in patients with chronic myeloid leukaemia who have maintained complete molecular remission for at least 2 years: the prospective, multicentre Stop Imatinib (STIM) trial. Lancet Oncol, 11, 1029-35.

Marin D, Ibrahim AR, Lucas C, et al (2012). Assessment of BCRABL1 transcript levels at 3 months is the only requirement for predicting outcome for patients with chronic myeloid leukemia treated with tyrosine kinase inhibitors. J Clin Oncol, 30, 232-8.

Nicolini FE, Turkina A, Shen ZX, et al (2012). An open-label, multicenter study of oral nilotinib in adult patient with imatinib-resistant or imatinib-intolerance Philadelphia chromosome positive chronic myeloid leukemia in the chronic phase, Expanding Nilotinib Access in Clinical Trial, ENACT. Cancer, 118, 118-26.

O'Brien SG, Guihot F, Larson RA, et al (2003). Imatinib compared with interferon and low-dose cytarabine for newly diagnosed chronic-phase chronic myeloid leukemia. N Engl J Med, 348, 994-1004.

O'Brien SG, Guilhot F, Goldman J, et al (2008). International randomized study of interferon versus STI571 (IRIS) 7-year follow-up: sustained survival, low rate of transformation and increased rate of major molecular response (MMR) in patients (pts) with newly diagnosed chronic myeloid leukemia in chronic phase (CML-CP) treated with imatinib (IM). Blood, 112, 76 .

Payandeh M, Sadeghi M, Sadeghi E (2015). Treatment and survival in patients with chronic myeloid leukemia in a chronic phase in the West of Iran. Asian Pac J Cancer Prev, 16, 7555-9.

Saglio G, Kim DW, Issaragrisil S, et al (2010). Nilotinib versus imatinib for newly diagnosed chronic myeloid leukemia. $N$ Engl J Med, 362, 2251-9.

Shah NP, Guilhot F,Cortes JE, et al (2014). Long-term outcome with dasatinib after imatinib failure in chronic-phase chronic myeloid leukemia: follow-up of a phase 3 study. Blood, 123, 2317-24.

Sokal JE, Cox EB, Baccarani M, et al (1984). Prognostic discrimination in "good-risk" chronic granulocytic leukemia.
Blood, 63, 789-99.

Testoni N, Marzocchi G, Luatti S, et al (2009). Chronic myeloid leukemia: a prospective comparison of interphase fluorescence in situ hybridization and chromosome banding analysis for the definition of complete cytogenetic response: a study of the GIMEMA CML WP. Blood, 114, 4939-43.

Vardiman JW, Melo JV, Baccarani M, et al (2008). Chronic myelogenous leukemia BCR-ABL1 positive. In: 'WHO classification of tumors of hematopoietic and lymphoid tissues', Eds Swerdlow SH, Campo E, Harris NL, et al. IARC, Lyon, 32-7.

Wang J, Shen ZX, Saglio G, et al (2015). Phase 3 study of nilotinib vs imatinib in Chinese patients with newly diagnosed chronic myeloid leukemia in chronic phase: ENESTchina. Blood, 125, 2771-8.

Yeung DT, Osborn MP, White DL, et al (2015). TIDEL-II: firstline use of imatinib in CML with early switch to nilotinib for failure to achieve time-dependent molecular targets. Blood, 125, 915-23. 